\title{
O ELETRENCEFALOGRAMA NAS HEMORRAGIAS UTERINAS DISFUNCIONAIS: AÇÃO DOS ESTROGENIOS POR VIA INTRAVENOSA
}

\author{
C. De Guarnieri Netto* \\ Luís Marques de Assis ** Laplace Pinto Vallada**
}

O aparecimento ou recrudescimento de crises epilépticas nos períodos menstruais tem sido referido por diversos autores. Laidlow ${ }^{6}$, verificando haver redução das crises epilépticas nas fases luteínicas e aumento irregular de tais crises antes, durante e após a menstruação, admitiu que a epilepsia catamenial pode ser devida à queda do nível de progesterona, que atuaria como anticonvulsionante. Ansell e Clarke 1, estudando 148 pacientes epilépticas, verificaram que em $63 \%$ delas havia recrudescimento das crises no periodo menstrual ou nas 48 horas anteriores; quanto ao pêso corporal, ao metabolismo do sódio e à quantidade total de água do organismo nos periodos menstruais, não foram assinaladas diferenças entre as pacientes epilépticas e não epilépticas. Barene e Gibbs ${ }^{2}$ fizeram estudos eletrencefalográficos durante o ciclo menstrual de jovens sadias, verificando não haver relação simples e rígida entre ciclo menstrual e atividade elétrica cerebral. Faure e colaboradores ${ }^{4,5}$ estudaram o eletrencefalograma na vigência de distúrbios menstruais (oligomenorréias, amenorréias, dismenorréias, hipermenorréias e metrorragia) e em relação a modificações hormônicas na mulher, estabelecendo verdadeira relação eletro-hormônica. Copelman 3, relacionando o nível hormônico com crises epilépticas, atribui a ocorrência destas à hiperestrogenia. Como Chavany e Fiamberti, Copelman admite que a acetilcolina desempenha papel fundamental no desencadeamento das crises epilépticas, pois a hiperfoliculinemia determina aumento do nível de acetilcolina no líquor e no sangue; nesse sentido Reynolds ${ }^{8}$ fêz experiências em cobaias, mostrando que a quantidade de acetilcolina no útero aumenta após injeção intravenosa de estrogênio. Baseado nestas verificações e na ação neutralizante da testosterona sôbre a hiperestrogenia, Copelman indica a testosterona no tratamento das epilepsias hiperestrogênicas. Logothetis $i$ estudou $o$ assunto mediante experiências em animais e em pacientes epilépticos; nos animais provocou lesōes corticais e depois produziu crises epilépticas usanđo o Premarin seja diretamente sôbre a lesão,

Trabalho apresentado no VI Congresso Brasileiro de Ginecologia (Salvador Bahia), em outubro (17 a 20) de 1960, como contribuição ao tema "Estado atual da terapêutica hormonal em Ginecologia": * Assistente extranumerário da Clínica Ginecológica da Fac. Med. da Univ. de São Paulo (Prof. José B. Medina); ** Da Clínica Neurológica da Fac. Med. da Univ. de Sāo Paulo (Prof. Adherbal Tolosa). 
seja por via intravenosa; nos pacientes epilépticos injetou Premarin, por via intravenosa, durante registro eletrencefalográfico, verificando o aparecimento de alterações de tipo epiléptico; em ambos os casos os estrogênios atuaram como hormônios epileptogênicos e ativadores do eletrencefalograma, levando o autor a admitir que o aparecimento ou a exacerbação das crises epilépticas nos períodos menstruais são devidos à ação dos estrogênios sôbre focos epileptogênicos préexistentes.

Levando em conta que no tratamento das hemorragias uterinas disfuncionais são largamente utilizados os estrogênios, estudamos um grupo de pacientes com tais disfunções, dando ênfase especial à verificação eletrencefalográfica antes, durante e após a injeção intravenosa de estrogênios.

\section{MATERIAL E MÉTODO}

Foram estudadas 10 pacientes internadas na vigência de crises hemorrágicas genitais. Em todos os casos, além de inquérito quanto aos antecedentes (familiares e pessoais) de natureza epiléptica, foi feito exame neurológico e exame do líqüido cefalorraquidiano obtido por punção suboccipital. Os exames eletrencefalográficos foram feitos em aparelho Grass de 8 canais, tendo sido usadas derivações unipolar e bipolares. Como ativante foi empregada a hiperpnéia em todos e a fotoestimulação em 6 casos. O registro foi feito antes, durante e até 30 minutos após a injeção intravenosa de estrogênios, variando o tempo de administraçāo entre 30 segundos e 4 minutos. Após a injeção foram utilizados os mesmos processos de ativação. Foi utilizado o Premarin (sulfato sódico de estrona) em uma única injeção de $20 \mathrm{mg}$. Foram feitas dosagens dos estrogênios urinários (método de Burmay) antes da administração do Premarin. Em 4 casos essa verificação foi feita após o tratamento hormonal ( 2 a 7 injeçōes intravenosas de $20 \mathrm{mg}$ de Premarin, com intervalo de 12 horas).

\section{RESULTADOS}

Dados clínico-neurológicos - Em 7 casos foram assinalados antecedentes (familiares ou pessoais) e/ou sintomas atuais de natureza provável ou certamente epiléptica. Em dois casos ocorreram convulsões na infância; em um ocorreu crise epiléptica precedendo a internação; dos 4 restantes, 2 apresentavam antecedentes familiares epilépticos. Em todos êsses pacientes foram assinalados sintomas de natureza provàvelmente epiléptica no passado ou no presente (crises de perda de fôlego, enxaqueca, sobressaltos noturnos, perdas de consciência de curta duração, agressividade, mêdo indefinido, sensação de estranheza, crises vertiginosas). O exame neurológico foi normal em tôdas as pacientes.

Dados eletrencefalográficos - Antes da administração do Premarin o eletrencefalograma foi normal em 5 casos e anormal em 5, com alterações assim constituídas: do tipo focal, com surtos de ondas de voltagem mais elevada que a. do ritmo dominante ( 3 casos), sendo o foco situado na região 
fronto-temporal esquerda (1) e temporal esquerda (2); do tipo difuso, com sensibilidade exagerada à hiperpnéia - surtos difusos de ondas lentas com voltagem mais elevada - em dois casos.

Em 8 casos (inclusive os 5 com eletrencefalograma normal) a administração de Premarin não provocou modificação dos traçados durante ou após a injeção. Nos dois casos de resposta exagerada à hiperpnéia, após injeção do Premarin houve melhora do traçado, caracterizada por resposta menos acentuada. A fotoestimulação não interferiu nos traçados eletrencefalográficos.

Correlação eletro-clínica - Dos três casos de hemorragia disfuncional não acompanhados de sintomas epilépticos e sem antecedentes epilépticos, o eletrencefalograma foi normal em dois e anormal em um (sensibilidade exagerada à hiperpnéia). Nos 7 casos com componente epiléptico, o eletrencefalograma resultou normal em 3 e anormal em 4 .

\begin{tabular}{|c|c|c|c|c|c|c|c|c|c|c|}
\hline Casos & 1 & 2 & 3 & 4 & 5 & 6 & 7 & 8 & 9 & 10 \\
\hline $\begin{array}{l}\text { Cenvulsões no } \\
\text { passado }\end{array}$ & & & & + & & + & & & & \\
\hline $\begin{array}{l}\text { Convulsōes no } \\
\text { presente }\end{array}$ & & + & & & & & & & & \\
\hline $\begin{array}{l}\text { Sintomas } \\
\text { epilépticos não } \\
\text { convulsivos no } \\
\text { passado }\end{array}$ & + & & & + & + & & + & & + & \\
\hline $\begin{array}{l}\text { Sintomas } \\
\text { epilépticos não } \\
\text { convulsivos no } \\
\text { presente }\end{array}$ & & & & & + & & & & + & \\
\hline $\begin{array}{l}\text { Epilepsia nos } \\
\text { familiares }\end{array}$ & & & & & & + & + & & + & \\
\hline ECG & $\mathrm{N}$ & $F$ & $\mathrm{~N}$ & $F$ & $\mathrm{~N}$ & $\mathrm{~N}$ & $F$ & $\mathrm{~N}$ & $\mathrm{D}$ & $\mathrm{D}$ \\
\hline
\end{tabular}

Quadro mostrando a correlação eletroclinica. Legenda: N, eletrencefalograma normal; $\mathrm{F}$, anormalidades focais; $\mathrm{D}$, anormalidades difusas.

Dados laboratoriais - O nivel dos estrogênios urinários antes da administração do Premarin manteve-se nos limites inferiores de normalidade (60 a 80 U.I.). Nos casos em que foi feita a verificação dêsses níveis após o tratamento, êles se mantiveram inalterados, independentemente da quantidade de estrogênio injetada.

o exame de líquor resultou normal em todos os casos. 


\section{COMENTÁRIOS}

É inegável que em grande número de pacientes epilépticos há aparecimento ou piora de crises nos períodos menstruais. $O$ ciclo menstrual determina profunda modificação hormônica da qual resultam distúrbios não sỏmente na esfera ginecológica, como na neuropsíquica. $O$ aparecimento ou agravamento de manifestações epilépticas durante o catamênio, ou imediatamente antes ou após, tem sido atribuído a diversos fatôres. A progesterona parece ter ação anticonvulsivante; êsse fato explicaria a diminuição da freqüência ou mesmo a não ocorrência de crises nas fases luteínicas. Por outro lado, os estrogênios atuam provocando aumento, no sangue e no liquor, de acetilcolina, substância que tem papel fundamental no desencadeamento de crises epilépticas; assim, os estrógenos teriam, indiretamente, ação convulsivante. A testoterona, tendo ação neutralizante sôbre a hiperestrogenia, foi preconizada no tratamento dos casos de epilepsia nos quais há excesso de hormônios estrogênicos.

Um dos tratamentos das hemorragias uterinas disfuncionais se baseia na administração intravenosa de estrogênios. Entretanto, essa medicação pode ocasionar libertação de acetilcolina, com desencadeamento de crises epilépticas em pacientes predispostas ou, pelo menos, com aparecimento ou acentuação de anormalidades eletrencefalográficas.

O estudo eletrencefalográfico de 10 pacientes com hemorragia uterina disfuncional mostrou que, dos 3 casos sem componente epiléptico algum, em um houve aumento da sensibilidade à hiperpnéia. Sintomas comiciais pré-existiam em 7 dos 10 casos. Levando em conta que se trata de pacientes que procuraram o hospital apenas devido à hemorragia uterina disfuncional, êsse dado sugere a existência de relação entre essa afecção e epilepsia. O fato de, dos 7 casos, $3(42,8 \%)$ apresentarem eletrencefalograma normal, não invalida essa suspeita, pois é sabido que, mesmo em pacientes comprovadamente epilépticos a positividade do exame eletrencefalográfico não é constante, principalmente quando se utiliza para ativação apenas a hiperpnéia e a fotoestimulação. Deve-se ressaltar, no entanto, que nos casos que estudamos os sintomas comiciais não mantinham relação com as menstruações, ou seja, não apareciam nem recrudesciam na época menstrual.

A análise dos eletrencefalogramas obtidos durante e após uma única injeção de $20 \mathrm{mg}$ de Premarin por via intravenosa, não confirmou os dados referidos na literatura. Pelo contrário, em dois de nossos casos houve discreta melhora do traçado eletrencefalográfico após injeção dos estrógenos. $O$ pequeno número de casos não permite qualquer conclusão a respeito. Apenas podemos dizer, tendo em vista os traçados eletrencefalográficos obtidos, que após injeção de $20 \mathrm{mg}$ de Premarin por via intravenosa não houve acentuação de anormalidades préexistentes, nem aparecimento de anormalidades nos casos de eletrencefalograma normal. A ação dêste preparado foi idêntica, tanto nos casos de hemorragia disfuncional pura como naqueles acompanhados de sintomas epilépticos. 
Em tôdas as pacientes os niveis estrogênicos foram baixos, tanto antes como após o tratamento pelo Premarin. Talvez a quantidade de estrógenos injetada fôsse insuficiente para determinar o aparecimento ou acentuação de anormalidades no eletrencefalograma.

\section{CONCLUSOEES}

1. De 10 casos de hemorragia disfuncional, 7 apresentaram antecedentes epilépticos e/ou sintomas de natureza provável ou certamente epiléptica; os sintomas não se relacionavam com os períodos menstruais.

2. Dos 7 casos com componente epiléptico, 4 apresentaram eletrencefalograma anormal.

3. Dos 3 casos de hemorragia disfuncional sem sintomas epilépticos, em apenas um o eletrencefalograma foi anormal.

4. Não houve piora do traçado eletrencefalográfico durante e após injeção intravenosa de $20 \mathrm{mg}$ de Premarin.

\section{RESUMO}

Em 10 pacientes do sexo feminino, portadoras de hemorragia uterina disfuncional, foi investigada a existência de manifestações epilépticas (clinicas e eletrencefalográficas) : dos 10 casos, 7 apresentavam antecedentes e/ou sintomas de natureza provável ou certamente epilépticos: o eletrencefalograma foi anormal em 5 casos.

Foi estudada a ação do Premarin por via intravenosa, na dose de 20 $\mathrm{mg}$, durante registro eletrencefalográfico. Não houve piora do traçado durante ou após a injeção.

\section{SUMMARY}

The electroencephalogram in functional uterine bleeding: action of the estrogens by intravenous infusion.

In 10 patients with functional uterine bleeding, the existence of epileptic manifestations (clinical and electroencephalographic) was investigated: 7 among the 10 cases had a previous history and/or symptoms of a nature that was probably or certainly epileptic; the electroencephalogram showed abnormalities in 5 cases.

The action of intravenous Premarin $(20 \mathrm{mg})$ was studied during electroencephalogram recording. There was no change in the normal electroencephalogram and in the patological ones there was not an increase of the abnormalities. 


\section{REFERENCIAS}

1. ANSELL, B.; CLARKE, E. - Epilepsy and menstruation: the role of water retention. Lancet, $2: 1232$, 1956. 2. BARENNE, D. D.; GIBBS, F. - Variations in the electroencephalogram during the menstrual cycle. Am. J. Obst. a. Gynec., 44:687, 1942. 3. COPELMAN, L. - Les effets remarquables de la testosterone dans le traitement de l'épilepsie hyperestrogenique ou hypoandrogenique. Proc. First Internat. Congr. Neurol. Sc., Bruxelas, 3:605, 1957. 4. FAURE, J.; LOISEAU, P. - Electroencephalogramme et troubles menstruels. Rev. Neurol., 95:525, 1956.5. FAURE, J.; MAJENDIE, J.; BOUSSEMART, E.; MASQUELIER, J. - Confrontation de l'EEG et de modifications hormonales chez la femme. Rev. Neurol., 94:168, 1956. 6. LAIDLOW, J. - Catamenial epilepsy. Lancet, $2: 1235,1956$. 7 . LOGOTHETIS, J.; HARNER, R.; MORREL, F.; TORRES, F. - The role of estrogens in catamenial exacerbation of epilepsy. Neurology, 9:352, 1959. 8. REYNOLDS, S. - The cholinergic action of oestrin. Science, 87:537, 1938.

Hospital das Clinicas - Faculdade de Medicina da Universidade de São Paulo -Caixa Postal 3461 - São Paulo, Brasil. 